\title{
Quantitative study of the effect of L-dopa and phenoxybenzamine on the rigidity of Parkinson's disease
}

\author{
COLIN J. ANDREWS ${ }^{1}$ AND DAVID BURKE ${ }^{2}$ \\ From the Division of Neurology, The Prince Henry Hospital and the School of Medicine, \\ University of New South Wales, Sydney, Australia
}

SUMMARY The static and dynamic components of the tonic stretch reflex and shortening reactions have been studied in biceps brachii, triceps, hamstrings, and quadriceps muscles of 19 patients with Parkinson's disease before and during L-dopa therapy. Clinical improvement during L-dopa administration correlated with a reduction in the dynamic component of the tonic stretch reflex in biceps, triceps, and quadriceps but not the hamstrings muscle, and with the static component of the tonic stretch reflex in biceps and triceps but not quadriceps and hamstrings muscles. The only shortening reaction consistently reduced during the L-dopa treatment period was the dynamic shortening reaction of the triceps muscle. The responses of the stretch reflexes to changes in muscle length were not altered by L-dopa therapy. In severely disabled patients the hamstrings and quadriceps stretch reflexes were maximal in a position of partial flexion of the knee joint and this response to muscle length was not altered in these patients despite a dramatic lessening of rigidity in some patients. Shortening reactions commonly remained in some patients after the stretch reflex of the antagonistic muscle was abolished by L-dopa therapy. The administration of phenoxybenzamine to patients being treated with L-dopa reduced the different components of rigidity more than L-dopa alone, and the responses were consistent with phenoxybenzamine suppressing predominantly the descending noradrenergic pathway.

The available evidence suggests that dopamine (DA) acts as a neurotransmitter at certain striatal synapses concerned with the mediation of inhibition in the nigroneostriatal pathway. Histochemical studies have demonstrated DA in cell bodies of neurones in the zona compacta of the substantia nigra, whereas caudate DA is concentrated within diffuse terminal varicosities distributed in close proximity to non-fluorescent caudate neurones (Andén, Carlsson, Dahlström, Fuxe, Hillarp, and Larsson, 1964; Ungerstedt, 1971). Electrical stimulation of the substantia nigra results in a depression of the firing rate of most neurones in the caudate nucleus (Connor, 1970); an increase in the homovanillic acid

1 Edwin and Daisy Street Fellow in Neurology.

2 Commonwealth Postgraduate Scholar and Adolph Basser Research Fellow in Neurology.

Address for reprints: Division of Neurology, The Prince Henry Hospital, Little Bay, N.S.W. 2036, Australia.
(HVA) concentration in the effluent from the anterior horn of the lateral ventricle (Portig and Vogt, 1969), and an increase in DA effluent collected by a push-pull cannula in the putamen (McLennan, 1965). Experimental lesions of the nigrostriatal pathway lead to depletion of striatal DA (Poirier and Sourkes, 1965) and a similar depletion of DA occurs in Parkinson's disease (Ehringer and Hornykiewicz, 1960). When DA is applied to the caudate nucleus by microelectrophoresis some $60 \%$ of neurones are inhibited (McLennan and York, 1967; Connor, 1968).

The popular explanation for the success of L-dopa therapy in Parkinson's disease is the replenishment of depleted stores of DA in the basal ganglia and reactivation of the nigrostriatal pathway. It is also likely that high concentrations of DA could influence the release, storage, 
and transport of other biogenic amines in the brain. The investigation of noradrenaline (NA) metabolism in the brain of man (after L-dopa administration) is difficult because vanilil mandelic acid (VMA) does not appear to be the major metabolite in the brain (Moir, Ashcroft, Crawford, Eccleston, and Guldberg, 1970), so that observations have largely been restricted to effects of L-dopa on the autonomic nervous system. The application of DA to the conjunctival sac causes pupillary dilatation which is probably due to release of noradrenaline (NA) (Spiers and Calne, 1969) and indeed pupillary dilatation occurs with systemic L-dopa therapy (Yahr, Duvoisin, Shear, Barrett, and Hoehn, 1969). Experimental work on animals indicates that DA may release NA from the descending bulbospinal noradrenergic system which originates in the medulla and traverses the spinal cord in the ventral and lateral quadrants (Carlsson, Falck, Fuxe, and Hillarp, 1964). In acute spinal cats this system can be activated by intravenous L-dopa, resulting in increased static fusimotor activity to both flexor and extensor muscles and increased dynamic fusimotor activity to extensor muscles, while dynamic fusimotor activity to flexor muscles is diminished (Bergmans and Grillner, 1968; Grillner, 1969). Baker and Anderson (1970a) were able to show an increase in amplitude of monosynaptic spikes and a decrease in stimulus-response latency after L-dopa in both flexor and extensor motor nuclei of acute spinal cats. This facilitation of monosynaptic transmission can be regarded as a measure of increased alpha motoneurone excitability. The function of the descending noradrenergic system is discussed in detail by Andrews, Neilson, and Knowles (1972). There is no agreement as to whether DA or NA is the effective neurotransmitter but Andén, Jukes, and Lundberg (1966) and Baker and Anderson (1970b) have shown that the effect is blocked by phenoxybenzamine.

In patients with the athetosis-spasticity syndrome, spasticity is reduced by phenoxybenzamine and the electromyographic analysis of the improvement is consistent with reduction by phenoxybenzamine of the activity of the descending noradrenergic system (Andrews et al., 1972). It is thus of interest to examine the dynamic and static components of the tonic stretch reflex in Parkinson's disease before and during the use of L-dopa, alone and in combination with phenoxybenzamine, and to compare the responses on the reflexes of flexor and extensor muscles.

\section{METHODS}

The 19 patients in this study were all participants in a clinical trial of L-dopa. Of the 19 patients aged from 49 to 75 years, six were female and 13 were male. Eight patients had previously been subjected to thalamotomy, unilateral in two and bilateral in six. The Webster rating scale (Webster, 1968) was used to score disability up to a maximum of $\mathbf{3 0}$ for the most severely affected patients. Clinical improvement has been expressed as a percentage of the original disability. The dosage of $\mathrm{L}$-dopa ranged from 1.0 to $8.0 \mathrm{~g}$ daily and, since improvement in rigidity may be delayed, recordings were made always after at least three months of treatment. In two patients, recordings were made at three, six, and 12 months. The principles of the electromyographic method of assessment have been described by Andrews, Burke, and Lance (1972).

The dynamic and static components of the tonio stretch reflex (TSR) were measured in the bicepso brachii, triceps, hamstrings, and quadriceps muscles 0 before treatment with L-dopa was started and at three, six, or 12 months of treatment. The line of besto fit of the relationship between velocity of stretch ando the height of the integrated electromyogram (EMG) was drawn and the threshold velocity, dynamic sensitivity, and the amplitude of the integrated EMG at $200^{\circ}$ of joint angle/sec was calculated from it to facilitate comparison of results at different treatment periods. The concepts of threshold velocity and dynamic sensitivity of the dynamic stretch reflex are explained in detail in an earlier paper (Andrews et al., 1973), and the same terminology is used in this paper.

The stretch reflex has been divided into dynamic and static components, being respectively the response evoked by the stretching movement, and that evoked by maintained static stretch. The shortening reaction has similar dynamic and static components. The relationship between the dynamic stretch reflex response and the velocity of stretch has been compared before and during treatment with L-dopa. The 'dynamic sensitivity' of the stretch reflex was calculated from the line of best fit of this relationship as the increase in integrated EMG for an increase in velocity of stretch by $100^{\circ} / \mathrm{sec}$, expressed as a percentage of that evoked at $400 \%$ sec. The EMG produced at $200 \% \mathrm{sec}$ was determined from the line of best fit of the integrated EMG : velocity relationship and has been used as a point of com- 


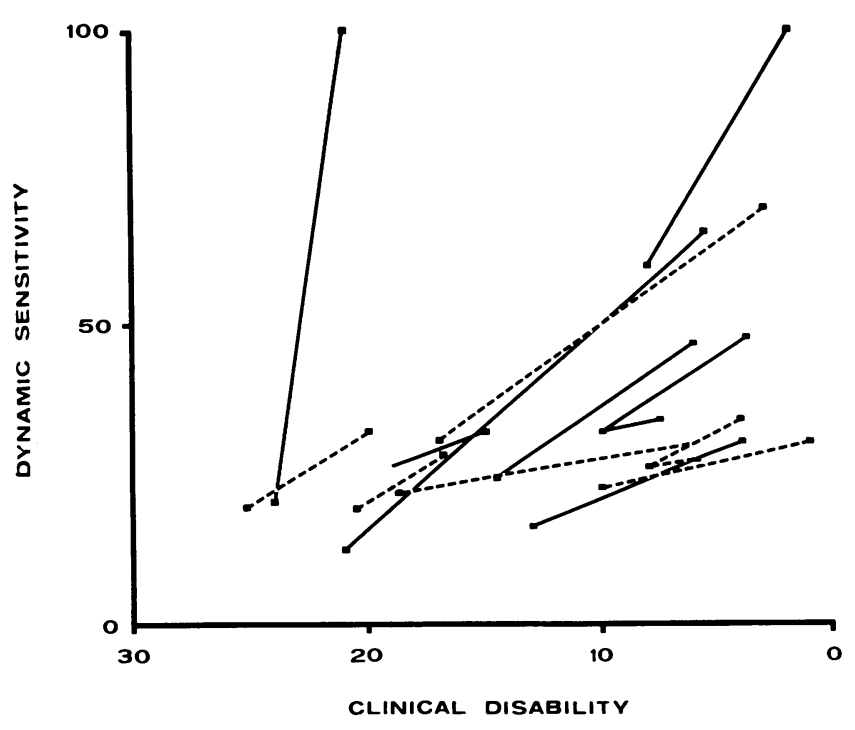

FIG. 1. The relationship of the dynamic sensitivity of the quadriceps stretch reflex to clinical improvement during L-dopa therapy. A lessening of clinical disability is associated with an increase in dynamic sensitivity of the stretch reflex. Broken line indicates patients with and continuous line patients without previous contralateral thalamotomy.

parison, since this velocity would be exceeded only rarely during routine clinical testing. Where appropriate, the intercept of the line of best fit of the EMG: velocity relationship has been taken as the threshold velocity of the dynamic stretch reflex.

The principle of the electromyographic method of assessment has been described by Andrews et al. (1973). In brief, the electromyogram evoked by passive manual movements was recorded using surface electrodes, and was integrated to provide a measure of reflex activity. Standard electrode placements were used so that for the two assessments comparable recordings could be obtained. The excursion of the limb at the appropriate joint was measured by a goniometer, the output of which was differentiated to provide a voltage proportional to angular velocity. The responses to muscle stretch and to muscle shortening were recorded in the biceps and triceps brachii, the hamstrings and quadriceps femoris muscles of the side most severely affected by rigidity.

After observations had been made on seven patients treated with L-dopa for three months or more, phenoxybenzamine $50 \mathrm{mg}$ was administered intravenously and EMG recordings were made 30 minutes afterwards. No alteration in the EMG was noticed for 10 minutes and the effect was most evident after 30 minutes when the final recordings were made. There was no significant change in blood pressure while the patients remained in the prone position but postural hypotension persisted for 24 hours after the injection, necessitating bed rest for this time. Phenoxybenzamine was administered orally to six patients in a nocturnal dose of $40 \mathrm{mg}$ in one patient and $20 \mathrm{mg}$ in five patients.

\section{RESULTS}

HAMSTRINGS AND QUADRICEPS MUSCLES The effects of L-dopa therapy on the dynamic stretch reflexes of the hamstrings and quadriceps muscles were studied in 18 patients. In the hamstrings, reflex EMG was of lower amplitude in 13 patients when on L-dopa therapy, but there was little change in the dynamic sensitivity of the hamstrings stretch reflex and there was no consistent relationship to the degree of clinical improvement. In the quadriceps muscle of nine patients, no EMG was recorded at $200 \% / \mathrm{sec}$ when on Ldopa therapy, although previously the reflex response had been prominent. The amplitude of EMG of the quadriceps muscle was reduced in a further four patients and was unaltered in five. The threshold velocity of the quadriceps stretch reflex increased in those who responded to L-dopa so that, although less EMG was recorded, the dynamic sensitivity of the stretch reflex increased. This change in the dynamic sensitivity of the quadriceps stretch reflex correlated with the degree of clinical improvement (Fig. 1). The absence of clinical improvement in 

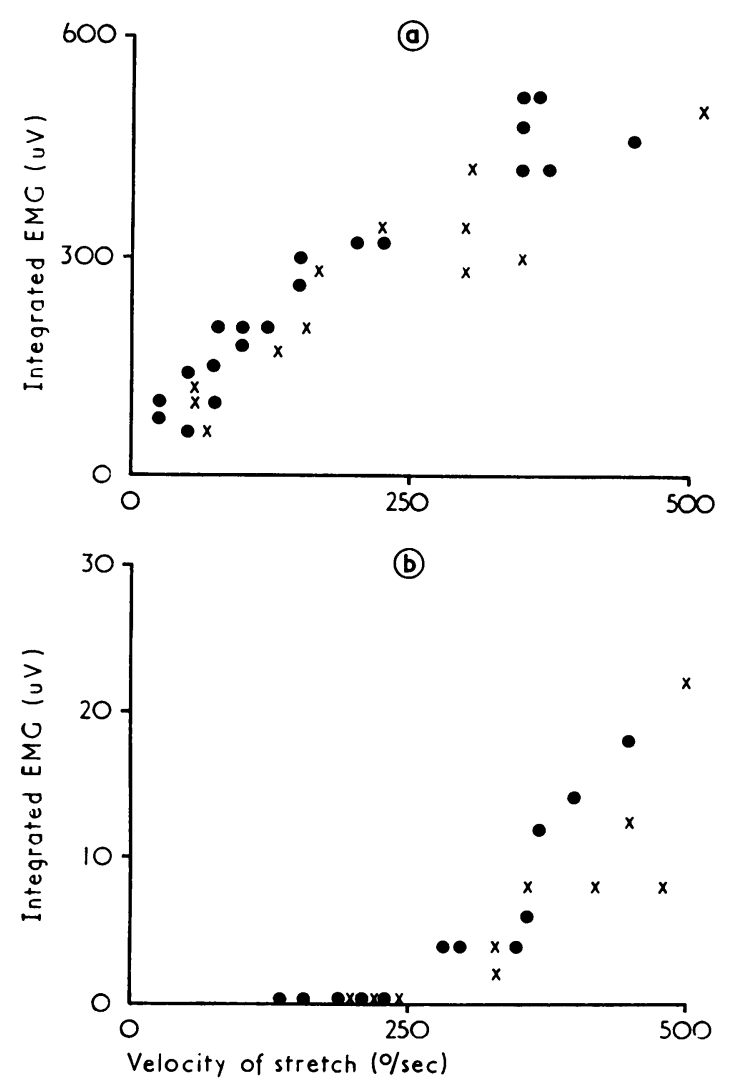

FIG. 2. Correlation of clinical response with EMG: the effect of increasing velocity of stretch on the dynamic stretch reflex (a) hamstrings and (b) quadriceps femoris muscles before and during L-dopa therapy in a patient who failed to improve clinically after six months. There was no significant difference between control and treatment periods. $=$ before, $\times=$ after L-dopa.

one patient was associated with an absence of measureable levels of HVA in the cerebrospinal fluid and the absence of any significant change of the dynamic stretch reflexes of hamstrings or quadriceps muscles (Fig. 2a and b).

The dynamic stretch reflex of the quadriceps muscle became maximal at the position of greatest stretch in 12 of 16 patients and short of the position of greatest stretch in four patients. These relationships were not altered by L-dopa therapy even though rigidity was greatly reduced by both clinical and electromyographic criteria. The hamstrings stretch reflex was maximal at the fully extended position of the knee joint in 11 of 16 patients and at a position of partial flexion in five patients. Again, these relationships were not altered by L-dopa except in one patient in whom the position of maximal EMG moved from $45^{\circ}$ flexion to the fully extended position of the knee joint.

A static stretch reflex was present in the quadriceps muscle of nine out of 16 patients, but was abolished by L-dopa therapy in only one patient. However, in the hamstrings muscle, a static reflex was recorded in 15 of 16 patients, and was abolished by L-dopa therapy in eight. There was no correlation between changes in the static reflexes and changes in the dynamic stretch reflexes (Fig. 3).

The dynamic shortening reaction of the quadriceps muscle was diminished during L-dopa therapy in nine out of 10 patients, and this change appeared to be related to a reduction in the antagonistic hamstrings stretch reflex. The dynamic shortening reaction of the hamstrings muscle was moderately reduced in five out of 10 patients during L-dopa therapy, but it was still recordable even when no stretch reflex response could be elicited from the antagonistic quadriceps muscle. A static shortening reaction was present in the quadriceps muscle of four patients

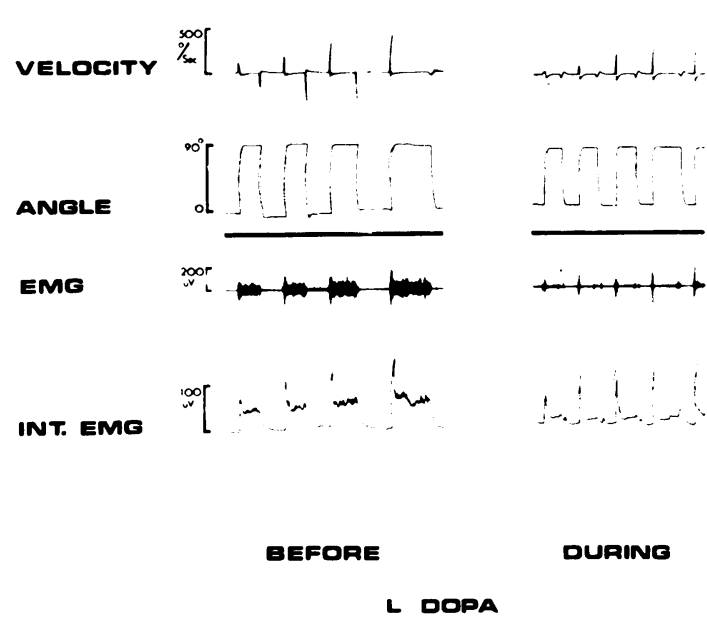

FIG. 3. The response of the hamstrings muscle to linear stretch before and during L-dopa therapy. The static stretch was markedly reduced during L-dopa therapy but no corresponding improvement occurred in the dynamic stretch reflex. 
and was abolished by L-dopa therapy in two. A static shortening reaction was recorded in the hamstrings muscle of four patients, and was abolished in three. Changes in the static shortening reflexes did not correlate with clinical improvement or with changes in the stretch reflex of the antagonist.

TRICEPS AND BICEPS MUSCLES The effect of L-dopa therapy on the dynamic stretch reflex of triceps and biceps muscles was studied in nine patients. In seven of the nine patients the amplitude of the triceps dynamic stretch reflex decreased on L-dopa therapy while the dynamic sensitivity was increased. In two patients with the inverse relationship, progression of their Parkinson's disease was suspected and this was verified on one patient on suspension of therapy. Decrease in amplitude and increase in dynamic sensitivity of the dynamic stretch reflex of the biceps muscle also correlated with clinical improvement in all patients except the sole patient with encephalitic Parkinsonism. The dynamic stretch reflexes of the biceps and triceps muscles were maximal at their most stretched position before and during L-dopa treatment.

The static reflex of the triceps muscle was present in five of nine patients examined before L-dopa therapy and was abolished in only one patient during treatment. In contrast the static stretch reflex was present in all nine patients in the biceps muscle at the fully stretched position and was abolished in six. Patients in whom the static stretch reflex remained suffered from severe rigidity and clinical disability which improved only slightly with treatment.

Decrease in the amplitude of the triceps dynamic shortening reaction correlated with clinical improvement. The dynamic shortening reaction increased in two of the seven patients examined, but in these two patients the disease was considered to have progressed while on L-dopa therapy. The marked reduction in the dynamic shortening reaction of the triceps and biceps muscles was not associated with a corresponding reduction in the stretch reflex of the antagonist. Indeed a dynamic shortening reaction was commonly present in the biceps muscle in the absence of a stretch reflex in the triceps. The triceps muscle was the only muscle of the four analysed that showed a correlation
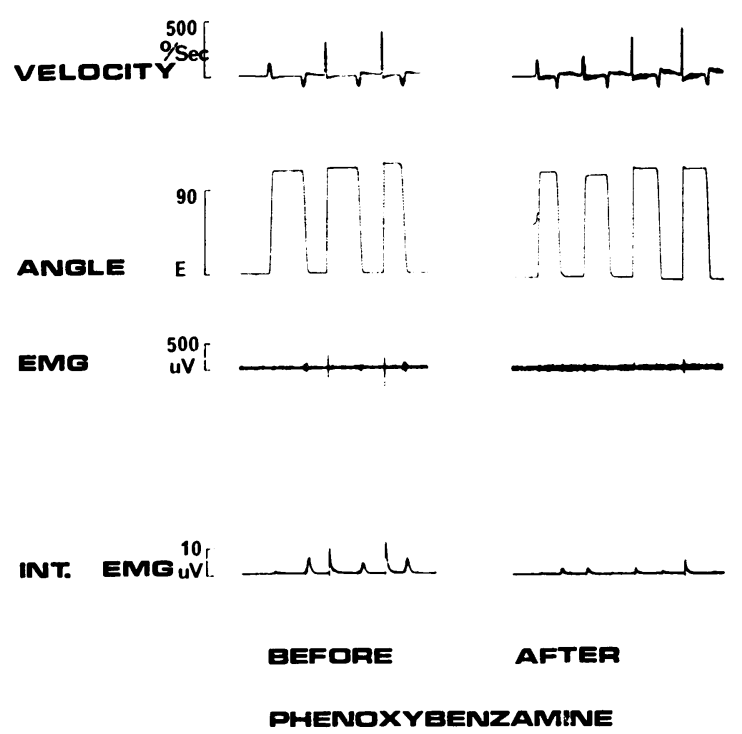

(a)

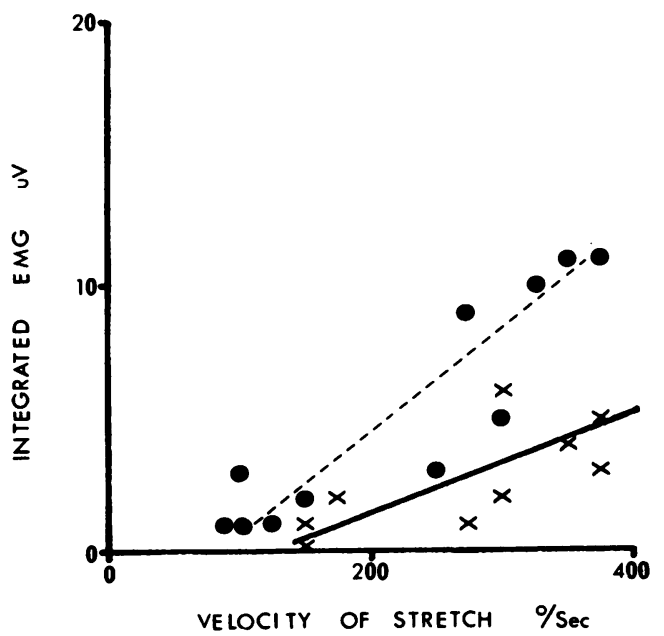

(b)

FIG. 4. (a) Reduction of the dynamic stretch reflex of quadriceps muscle by intravenous phenoxybenzamine $50 \mathrm{mg}$ in a patient receiving $\mathrm{L}$-dopa therapy. (b) $A$ graph showing a decrease in the reflex response at all velocities of stretch after phenoxybenzamine administration. $\quad L-O=$ dopa $. \quad \times=L-d o p a+$ phenoxybenzamine.

between clinical improvement and reduction in the dynamic shortening reaction. The static shortening reaction of both muscles usually 
persisted even in patients with a good clinical response to administration of $\mathrm{L}$-dopa.

ADMINISTRATION OF PHENOXYBENZAMINE DURING L-DOPA THERAPY After phenoxybenzamine 50 mg was given intravenously the dynamic stretch reflex of the quadriceps muscle was reduced in all six patients examined but the dynamic sensitivity was altered only slightly (Fig. 4a and $b$, and Table). On the other hand, the amplitude of the hamstrings dynamic tonic stretch reflex increased in two of the seven patients at $200^{\circ} / \mathrm{sec}$ angular velocity and the dynamic sensitivity increased in all patients (Fig. 5a and b, and Table). At high velocities of stretch the hamstrings stretch reflex was usually greater and in two patients it was also of greater amplitude at $200^{\circ} / \mathrm{sec}$.

TABLE

DYNAMIC SENSITIVITY

\begin{tabular}{|c|c|c|c|c|c|}
\hline \multirow[t]{3}{*}{ Patient } & \multicolumn{4}{|c|}{ Before and after phenoxybenzamine } & \multirow{3}{*}{$\begin{array}{c}L \text {-dopa } \\
(g \text { daily })\end{array}$} \\
\hline & \multicolumn{2}{|c|}{ Quadriceps } & \multicolumn{2}{|c|}{ Hamstrings } & \\
\hline & Before & After & Before & After & \\
\hline $1 *$ & 32 & 33 & 0 & 27 & $2 \cdot 0$ \\
\hline $2^{*}$ & - & - & 18 & 32 & $7 \cdot 5$ \\
\hline $3^{*}$ & 27 & 29 & 19 & 26 & 5.0 \\
\hline 4 & 100 & 100 & 21 & 50 & 5.0 \\
\hline 5 & 66 & 66 & 0 & 13 & 3.0 \\
\hline 6 & 100 & 125 & 18 & 27 & $1 \cdot 5$ \\
\hline 7 & 25 & 31 & 33 & 66 & 6.0 \\
\hline
\end{tabular}

* Thalamotomy.

The static reflex was reduced by phenoxybenzamine and was abolished in one patient in the hamstrings and in two patients in the quadriceps muscle. The response of the dynamic and static stretch reflexes to varying joint position remained unaltered in all patients after phenoxybenzamine. The dynamic shortening reaction in quadriceps and hamstrings muscles improved in all patients as judged by the increase in dynamic sensitivity and reduction in the amplitude of the integrated EMG at $200^{\circ} / \mathrm{sec}$. The static shortening response was abolished in one of the two patients in whom it was present in the hamstrings muscle.

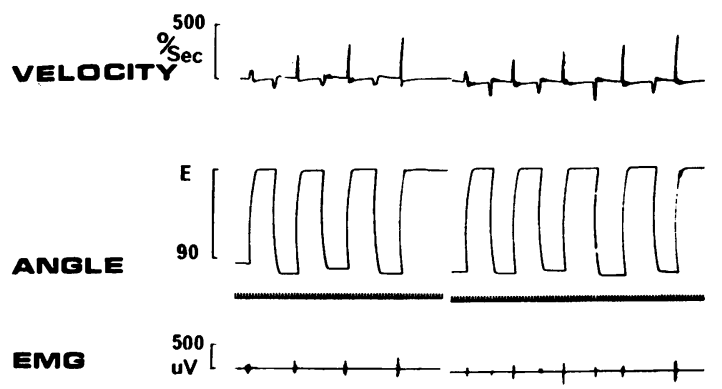

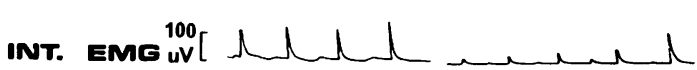

BEFORE AFTER

PHENOXYEENZAMLNE

(a)

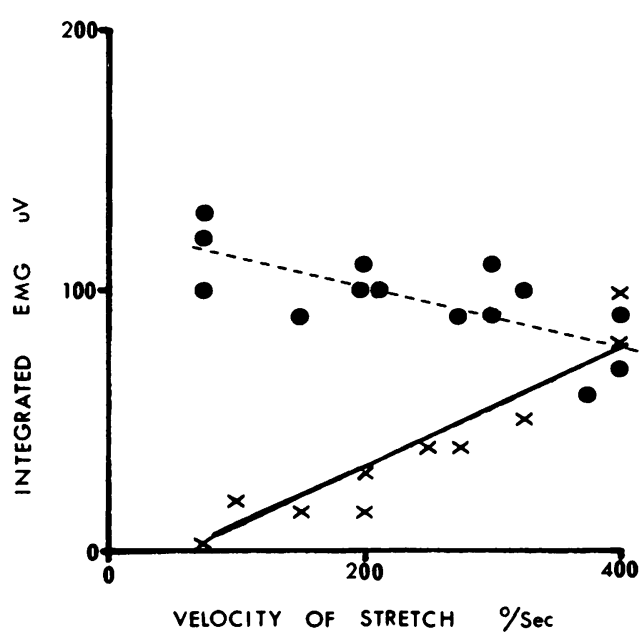

(b)

FIG. 5. (a) Reduction of the reflex response of the hamstrings muscle to linear stretch after phenoxybenzamine administration with relative increase in dynamic sensitivity. (b) $A$ graph of the relationship between the integrated EMG of the hamstrings stretch reflex and velocity of stretch showing that the reflex is predominantly suppressed at low velocities of stretch after phenoxybenzamine administration. $=$ L-dopa. $\times=L$-dopa + phenoxybenzamine. 
In six patients to whom phenoxybenzamine was administered orally, three had mild subjective and objective improvement in gait. Hand function was not improved. In one patient on the highest tolerated dose electromyographic studies showed changes similar to those with intravenous phenoxybenzamine. In the remainder, satisfactory doses of phenoxybenzamine could not be reached because of postural hypotension. Patients with pre-existing postural hypotension on L-dopa required only a small dose of phenoxybenzamine to aggravate this side-effect.

\section{DISCUSSION}

Clinical improvement during L-dopa therapy has been found to correlate with reduction in the dynamic component of the tonic stretch reflex of quadriceps, triceps, and biceps but not the hamstrings muscles and with the static component of the tonic stretch reflex in biceps and triceps but not the quadriceps and hamstrings muscles. The reduction of the dynamic shortening reaction of the triceps muscle was the only shortening reaction of the four muscles examined which was found to correlate with clinical improvement. When phenoxybenzamine was administered together with L-dopa, the dynamic tonic stretch was reduced at high velocities of stretch in the quadriceps and increased in the hamstrings muscle, the dynamic sensitivity remained unchanged in the quadriceps and increased in the hamstrings muscle. The static reflexes and shortening reactions were reduced during phenoxybenzamine administration. There is evidence that the rigidity of Parkinson's disease depends upon excessive supraspinal drive of alpha motoneurones (Yap, 1967), as well as gamma motoneurones (Rushworth, 1961), both static (Hagbarth, Hongell, and Wallin, 1970) and dynamic (Dietrichson, 1971). Dynamic tonic stretch reflexes thus depend upon both alpha motoneurone and dynamic fusimotor activity. The dynamic tonic stretch reflexes at low velocities of stretch will also be influenced by static fusimotor activity. The dynamic sensitivity of the quadriceps, triceps, and biceps muscle, has been shown to increase with clinical improvement in patients treated with L-dopa, whereas the hamstrings dynamic tonic stretch reflex failed to show this response. An explanation for this discrepancy is the activation of the descending noradrenergic bulbospinal system by L-dopa administration. The activation of this system would be expected to result in an increase in alpha motoneurone excitability and a decrease in dynamic fusimotor drive and therefore to reduce the dynamic sensitivity of the hamstrings stretch reflex. The increase in the dynamic sensitivity of the hamstrings dynamic tonic stretch reflex in all patients examined and its augmentation at high velocities of stretch in the majority of patients when L-dopa and phenoxybenzamine were used concomitantly suggests that L-dopa may have activated this system. The correlation of improvement of static stretch reflexes with clinical improvement in the triceps and biceps and not in the quadriceps and hamstrings muscles is probably due to the larger representation of the descending noradrenergic system on the lower limbs (Andén et al., 1966). Recently fusaric (5butylpicolinic) acid, a potent inhibitor of dopamine betahydroxylase has been found effective in the treatment of Parkinson's disease when used together with L-dopa. The concentration of cerebral serotonin in rats injected with fusaric acid increases, whereas cerebral noradrenaline decreases (Hidaka, 1971). When fusaric acid and L-dopa were administered sequentially, brain serotonin and noradrenaline levels remained similar to uninjected animals. Thus, fusaric acid prevented the decrease of brain serotonin caused by administration of L-dopa, and L-dopa prevented the depletion of noradrenaline by fusaric acid. Hidaka postulates that fusaric acid is effective by preventing depletion of serotonin in patients with Parkinsonism treated with L-dopa. However, the precursor of serotonin, 5-hydroxytryptophan, when administered in large oral doses with a peripheral decarboxylase inhibitor to patients with idiopathic Parkinson's disease, caused a clinical deterioration (Chase, 1970). An alternative explanation to that of Hidaka is that fusaric acid prevents the increase in noradrenaline in the brain-stem that follows the administration of L-dopa (Reis, Moorhead, and Melino, 1970), causing a response similar to the blocking of noradrenergic receptors with phenoxybenzamine. It is unfortunate that phenoxybenzamine when used together with L-dopa nearly always causes severe postural hypotension. 
However, its beneficial effects in overcoming the undesirable effects of L-dopa on the descending noradrenergic bulbospinal system may well be simulated by fusaric acid.

In severe cases of Parkinson's disease the hamstrings and quadriceps dynamic and static stretch reflexes are maximal in a position of partial knee flexion and this response was unaltered by L-dopa therapy, whether given alone or in combination with phenoxybenzamine, even though the rigidity was markedly improved in some patients. Clinical improvement was associated with an increase in dynamic spindle sensitivity so that the dynamic stretch reflexes resembled those of patients with mild untreated Parkinson's disease. Although the response to velocity was restored by L-dopa therapy, a similar reversal of the response to changing muscle length did not occur. This is presumably a manifestation of progressive flexion dystonia and may be useful as a criterion for the progression of Parkinson's disease which is marked in other respects by the response to L-dopa.

The authors wish to thank Associate Professor J. W. Lance for his encouragement and constructive criticism. Illustrations were photographed by the Department of Medical Illustration, University of New South Wales. This study was carried out with the financial assistance of the National Health and Medical Research Council of Australia, the Adolph Basser Trust, and Mr. and Mrs. Edwin Street.

\section{REFERENCES}

Andén, N.-E., Carlsson, A., Dahlström, A., Fuxe, K., Hillarp, N.-Å., and Larsson, K. (1964). Demonstration and mapping out of nigro-neostriatal dopamine neurons. Life Sciences, 3, 523-530.

Andén, N.-E., Jukes, M. G. M., and Lundberg, A. (1966). The effect of DOPA on the spinal cord. 2. A pharmacological analysis. Acta Physiologica Scandinavica, 67, 387-397.

Andrews, C. J., Burke, D., and Lance, J. W. (1973). The responses to muscle stretch and shortening in Parkinsonian rigidity. Brain. (In press.)

Andrews, C. J., Neilson, P., and Knowles, L. (1973). An electromyographic study of the rigidospasticity of athetosis. Journal of Neurology, Neurosurgery, and Psychiatry, 36, 94-103.

Baker, R. G., and Anderson, E. G. (1970a). The effects of L-3, 4-dihydroxyphenylalanine on spinal reflex activity. Journal of Pharmacology and Experimental Therapeutics, 173, 212-223.

Baker, R. G., and Anderson, E. G. (1970b). The antagonism of the effects of L-3, 4-dihydroxyphenylalanine on spinal reflexes by adrenergic blocking agents. Journal of Pharmacology and Experimental Therapeutics, 173, 224-231.

Bergmans, J., and Grillner, S. (1968). Changes in dynamic sensitivity of primary endings of muscle spindle afferents induced by DOPA. Acta Physiologica Scandinavica, 74, 629-636.

Carlsson, A., Falck, B., Fuxe, K., and Hillarp, N.-Å. (1964). Cellular localization of monoamines in the spinal cord. Acta Physiologica Scandinavica, 60, 112-119.

Chase, T. N. (1970). 5-Hydroxytryptophan in Parkinsonism. Lancet, 2, 1029-1030.

Connor, J. D. (1968b). Caudate unit responses to nigral stimuli: evidence for a possible nigro-neostriatal pathway. Science, 160, 899-900.

Connor, J. D. (1970). Caudate nucleus neurones: correlation of the effects of substantia nigra stimulation with iontophoretic dopamine. Journal of Physiology, 208, 691-703.

Dietrichson, P. (1971). Phasic ankle reflex in spasticity and Parkinsonian rigidity. Acta Neurologica Scandinavica, 47, 22-51.

Ehringer, H., and Hornykiewicz, O. (1960). Verteilung von Noradrenalin und Dopamin (3-Hydroxytyramin) im Gehirn des Menschen. Klinische Wochenschrift, 38, 12361239.

Grillner, S. (1969). The influence of DOPA on the static and the dynamic fusimotor activity to the triceps surae of the spinal cat. Acta Physiologica Scandinavica, 77, 490-509.

Hagbarth, K.-E., Hongell, A., and Wallin, G. (1970) Parkinson's disease: afferent muscle nerve activity in rigid patients. A preliminary report. Acta Societatio Medicorum Upsaliensis, 75, 70-76.

Hidaka, H. (1971). Fusaric (5-butylpicolinic) acid, an inhibitor of dopamine $\beta$-hydroxylase, affects serotonin and noradrenaline. Nature, 231, 54-55.

McLennan, H. (1965). The release of dopamine from the putamen. Experientia, 21, 725-726.

McLennan, H., and York, D. H. (1967). The action of dopamine on neurones of the caudate nucleus. Journal of Physiology, 189, 393-402.

Moir, A. T. B., Ashcroft, G. W., Crawford, T. B. B., Eccleston, D., and Guldberg, H. C. (1970). Cerebral metabolites in cerebrospinal fluid as a biochemical approach to the brain. Brain, 93, 357-368.

Poirier, L. J., and Sourkes, T. L. (1965). Influence of the substantia nigra on the catecholamine content of the striatum. Brain, 88, 181-192.

Portig, P. J., and Vogt, M. (1969). Release into the cerebral ventricles of substances with possible transmitter function in the caudate nucleus. Journal of Physiology, 204, 687-715.

Reis, D. J., Moorhead, D. T., II, and Melino, N. (1970). Dopa-induced excitement in the cat. Its relationship to brain norepinephrine concentrations. Archives of Neurology, 22, 31-39.

Rushworth, G. (1961). The gamma system in parkinsonism. International Journal of Neurology, 2, 34-50.

Spiers, A. S. D., and Calne, D. B. (1969). Action of dopamine on the human iris. British Medical Journal, 4, 333-335.

Ungerstedt, U. (1971). Stereotaxic mapping of the monoamine pathways in the rat brain. Acta Physiologica Scandinavica, Suppl. 367, 1-48.

Webster, D. D. (1968). Critical analysis of the disability in Parkinson's disease. Modern Treatment, 5, 257-282.

Yahr, M. D., Duvoisin, R. C., Shear, M. J., Barrett, R. E., and Hoehn, M. M. (1969). Treatment of Parkinsonism with levodopa. Archives of Neurology, 21, 343-354.

Yap, C. B. (1967). Spinal segmental and long-loop reflexes on spinal motoneurone excitability in spasticity and rigidity. Brain, 90, 887-896. 